\title{
Remember/Know judgments in cognitive neuroscience: An illustration of the underrepresented point of view
}

\author{
John T. Wixted ${ }^{1}$ \\ Department of Psychology, University of California, San Diego, La Jolla, California 92093-0109, USA
}

\begin{abstract}
The Remember/Know procedure is designed to easily measure two memory processes (recollection and familiarity) that are thought to serve as the basis for recognition memory decisions. This procedure is widely used in both neuroimaging studies and in studies involving amnesic patients in an effort to identify the brain structures that subserve these two memory processes. An alternative interpretation of this procedure, based on signal-detection theory, holds that Remember judgments and Know judgments are not "process pure" and are instead indicative of different degrees of memory strength (e.g., high degrees of recollection and familiarity vs. low degrees of recollection and familiarity, respectively). In the discipline of experimental psychology, the signal-detection view is widely regarded as a viable (or even preferable) alternative to the process-pure view. In the neuroscience literature, by contrast, the signal-detection interpretation is rarely given serious (or even any) consideration. Because conclusions about the neuroanatomical basis of recollection and familiarity are dependent on one specific interpretation of the Remember/Know procedure, ignoring a viable alternative interpretation may be counterproductive.
\end{abstract}

Recognition memory procedures are often used to investigate the neuroanatomical basis of declarative memory. In a typical recognition memory test, target items that previously appeared on a list are randomly intermixed with lures that did not, and these items are individually presented to the subject for an Old/New recognition decision. Dual process theories of recognition memory hold that items can be recognized as being old based either on recollection or on familiarity (Mandler 1980). Recollection involves consciously retrieving contextual details that were associated with the item at the time of encoding (e.g., remembering that "zoo" was presented as the second item in the list and, at the time, prompted thoughts about giraffes), whereas familiarity involves confidently knowing that the item was on the list even though no specific information about its prior occurrence can be recalled.

Interest in identifying the neural correlates of these two memory processes has greatly increased in recent years, and one behavioral method that is widely used to facilitate that effort is the Remember/Know procedure. Although originally intended to distinguish between episodic and semantic memory (Tulving 1985), the Remember/Know procedure is now widely used instead to distinguish between recollection and familiarity. The procedure involves asking individuals to indicate, for each item that is declared to be old, whether their decision was based on the recollection of encoding details (in which case they are instructed to say "Remember") or on a recollection-free feeling of familiarity (in which case they are instructed to say "Know").

In recent years, this convenient technique has been used in many neuroimaging studies and in many lesion studies in an effort to identify brain structures that underlie recognition memory processes. Differential neural activity associated with Remember and Know judgments or differential deficits in those judgments exhibited by amnesic patients have been interpreted to mean that different brain structures subserve the recollection and

\footnotetext{
'Corresponding author.
}

E-mail jwixted@ucsd.edu; fax (858) 534-7190.

Article is online at http://www.learnmem.org/cgi/doi/10.1101//m.1312809. familiarity processes (e.g., Eldridge et al. 2000, 2005; Holdstock et al. 2002, 2005; Moscovitch and McAndrews 2002; Yonelinas et al. 2002; Aggleton et al. 2005; Gonsalves et al. 2005; Uncapher and Rugg 2005; Verfaellie et al. 2008).

An alternative interpretation of Remember/Know judgments, originally proposed by Donaldson (1996), is based on another long-standing model of recognition memory known as signaldetection theory (Egan 1958). This model holds that targets and lures on a recognition test are associated with overlapping distributions of memory strength, with the average strength of the targets being greater than that of the lures. The distance between the means of two distributions (known as $d^{\prime}$ ) represents the ability of a participant (or a group of participants) to discriminate targets from lures. The larger the $d$, the less the two distributions overlap and the better the discrimination ability is. According to this model, any test item that generates a memory strength exceeding a criterion value is declared to be Old, but otherwise it is declared to be New (as illustrated in Figure 1A). The farther an item's memory strength falls above or below the criterion, the more confident the subject is that the item is old or new (and the faster the decision will be). Often, confidence ratings are made on a sixpoint scale (e.g., 1 = Sure New, 2 = Probably New, 3 = Maybe New, 4 = Maybe Old, $5=$ Probably Old, and $6=$ Sure Old). These confidence ratings are assumed to be based on additional confidence criteria situated along the memory strength axis, as illustrated by the dashed vertical lines in Figure 1A. An item with a memory strength that exceeds the rightmost criterion receives a confidence rating of 6 (Sure Old). An item with a memory strength that falls between the two rightmost criteria receives a confidence rating of 5 (Probably Old), and so on.

The signal-detection account assumes that recognition memory decisions are based on a singular memory strength variable, which appears to place it into conflict with the widely accepted dual-process theory of recognition. However, while signal-detection theory is, indeed, compatible with a single process account (i.e., memory strength could be based on one process), it is also compatible with a dual-process account, one that holds that recollection 

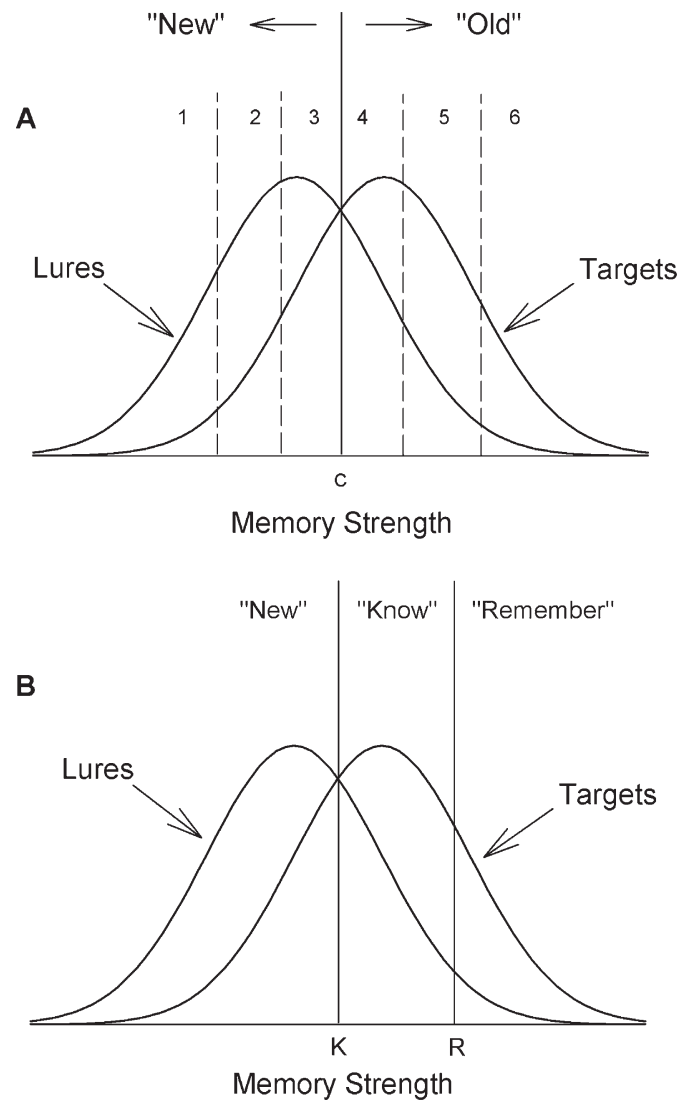

Figure 1. Illustrations of $(A)$ the standard signal-detection model of recognition memory and $(B)$ the signal-detection interpretation of Remember/Know judgments. The target distribution represents the memory strength values associated with the items that appeared in the list, and the lure distribution represents the baseline memory strength of the items that did not. In $A$, c denotes the criterion strength above which an item is declared to be Old, and the numbers $1-6$ correspond to confidence ratings ( $1=$ Sure New, $6=$ Sure Old). In $B, \mathrm{~K}$ and $\mathrm{R}$ denote the criterion strengths above which test items are given Know or Remember judgments, respectively.

and familiarity are both continuous processes that are aggregated to determine the memory strength of a particular test item (Wixted 2007). Thus, adopting a signal-detection view of Remember/Know judgments does not entail rejecting the dual-process view of recognition memory. Instead, it entails rejecting the idea that Remember responses are based on recollection, and Know responses are based on familiarity (i.e., that Remember and Know judgments are "process pure").

Donaldson (1996) first proposed that Remember and Know judgments simply reflect different degrees of memory strength and, therefore, different levels of confidence. According to his account, as illustrated in Figure 1B, Remember judgments are made to items that exceed a high memory-strength criterion (labeled R), whereas Know judgments are made to items that only exceed a lower criterion (labeled $\mathrm{K}$ ). The $\mathrm{K}$ criterion is equivalent to the old/new decision criterion (c) in Figure 1A, whereas the R criterion is placed at a higher point on the memory strength axis. The Remember hit rate corresponds to the proportion of the target distribution that exceeds the $\mathrm{R}$ criterion, and the Know hit rate corresponds to the proportion of the target distribution that falls between the $\mathrm{K}$ and $\mathrm{R}$ criteria (with the overall hit rate being the sum of those two values). The false alarm rates associated with Remember and Know judgments correspond to the proportion of the lure distribution that exceeds the $\mathrm{R}$ criterion and that falls between the $\mathrm{R}$ and $\mathrm{K}$ criteria, respectively.

Donaldson's (1996) signal-detection interpretation of Remember/Know judgments offers a natural account of several findings that are hard to reconcile with the process-pure interpretation. For example, much evidence suggests that the recollection process is slower than the familiarity process (Mandler 1980; Yonelinas 2002). Thus, according to the process-pure interpretation of Remember/Know judgments, the natural prediction is that Know judgments (which are assumed to reflect familiarity) should be made more quickly than Remember judgments (which are assumed to reflect recollection). However, the opposite result is reliably observed (Dewhurst et al. 2006; Rotello and Zeng 2008). This finding is easily understood in light of the signal-detection interpretation, which assumes that Remember responses reflect high-confidence decisions (based on items with a memory strength far above the old/new criterion), whereas Know responses reflect lower confidence decisions (based on items with a memory strength closer to the old/new criterion). In contrast to the straightforward explanation offered by signal-detection theory, the processpure interpretation must assume that additional variables influence Remember/Know reaction times. More specifically, Yonelinas (2002) argued that the unexpected reaction time pattern is an artifact of standard Remember/Know instructions, which specify that a Know judgment should be made only when an item is not recollected. Such instructions may encourage participants to withhold responding on the basis of familiarity until they are sure that recollection will not succeed. Dewhurst et al. (2006) directly tested this idea by eliminating any incentive to wait for recollection, but reaction times were still faster for Remember judgments than for Know judgments. In light of these results, they rejected the process-pure interpretation of Remember/Know judgments and noted that their results are compatible with the signal-detection interpretation.

In the experimental psychology literature, the signal-detection interpretation of Remember/Know judgments is widely understood to be a strong competitor to the process-pure interpretation (e.g., Hirshman and Henzler 1998; Inoue and Bellezza 1998; Dunn 2004, 2008; Wixted and Stretch 2004; Benjamin 2005; Rotello et al. 2005; Algarabel and Pitarque 2007; Rotello and Zeng 2008; Starns and Ratcliff 2008). Indeed, it may be no exaggeration to say that it is now the dominant account. In the neuroscience literature, by contrast, the process-pure interpretation is almost exclusively applied. One reason for the discrepancy may be that a clear illustration of the viability and applicability of the signal-detection interpretation is generally lacking in the pages of neuroscience journals. Most studies in the neuroscience literature that make use of the Remember/Know procedure to investigate recollection and familiarity fail to even mention the signal-detection interpretation. The few studies that do briefly mention it do not explain it in enough detail to be understood by those who are not already intimately familiar with it. Moreover, these studies do not adequately test the ability of signal detection theory to account for the data that are invariably interpreted in terms of the process-pure view of Remember/Know judgments.

In what follows, I present the signal-detection interpretation of one finding from the experimental psychology literature (a finding that, on the surface, appears to weigh strongly against the signal-detection account) and one finding from the neuroscience literature (the interpretation of which is altered if the signaldetection interpretation is correct). My purpose in presenting these illustrations is to encourage a more serious consideration of the signal-detection interpretation in studies that rely on the Remember/Know procedure to identify the brain basis of recollection and familiarity. 
The signal-detection account holds that Remember/Know judgments are tantamount to confidence ratings, with Remember judgments reflecting relatively high confidence (associated with strong memory) and Know judgments reflecting relatively low confidence (associated with weaker memory). One of the most highly cited Remember/Know studies in the experimental psychology literature directly tested and appeared to convincingly refute this idea. Gardiner and Java (1990) presented participants with a list of nonwords to memorize. In one experiment, they asked participants to make Remember/Know judgments following each Old decision. In another, they instead asked for confidence ratings (Sure/Unsure). If one assumes that Remember judgments are similar to Sure responses and that Know judgments are similar to Unsure responses (as signal detection theory seems to do), then the results should be the same in both cases. However, the results ran contrary to this prediction. Specifically, participants made more Know judgments than Remember judgments to target items (i.e., the Know hit rate was higher than the Remember hit rate), but they made more Sure responses than Unsure responses to target items. Figure 2 reproduces the findings of interest. If Remember/Know judgments are tantamount to Sure/Unsure judgments, how could this dissociation have been observed?

Despite appearances to the contrary, the signal-detection interpretation of these findings is straightforward (as noted by Inoue and Bellezza [1998] and Dunn [2004]). Figure 3A shows the simplest signal-detection illustration of the results from the Remember/Know condition, and Figure 3B shows the corresponding illustration for the Sure/Unsure condition. The overall hit and false alarm rates in the Remember/Know condition were 0.48 and 0.14 , respectively, which yields a $d^{\prime}$ of $\sim 1.0$. That is, the distance between the mean of the target distribution and the mean of the
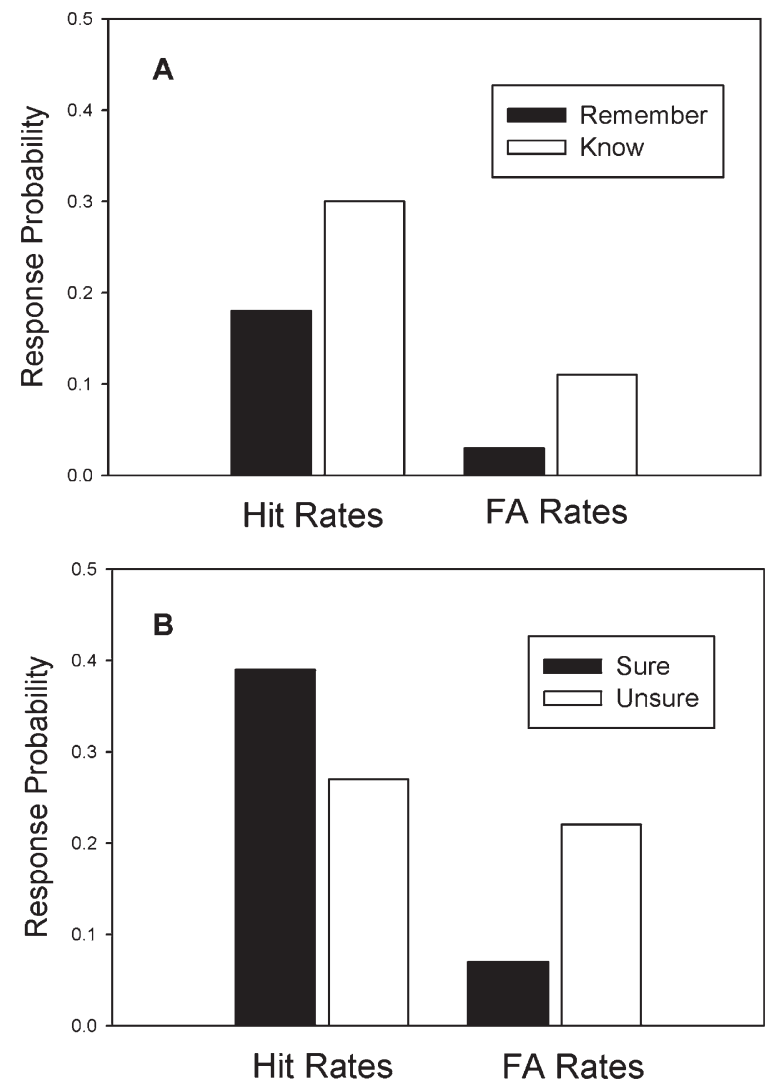

Figure 2. (A) Reproduction of nonword Remember/Know data and (B) the Sure/Unsure data, reported by Gardiner and Java (1990).

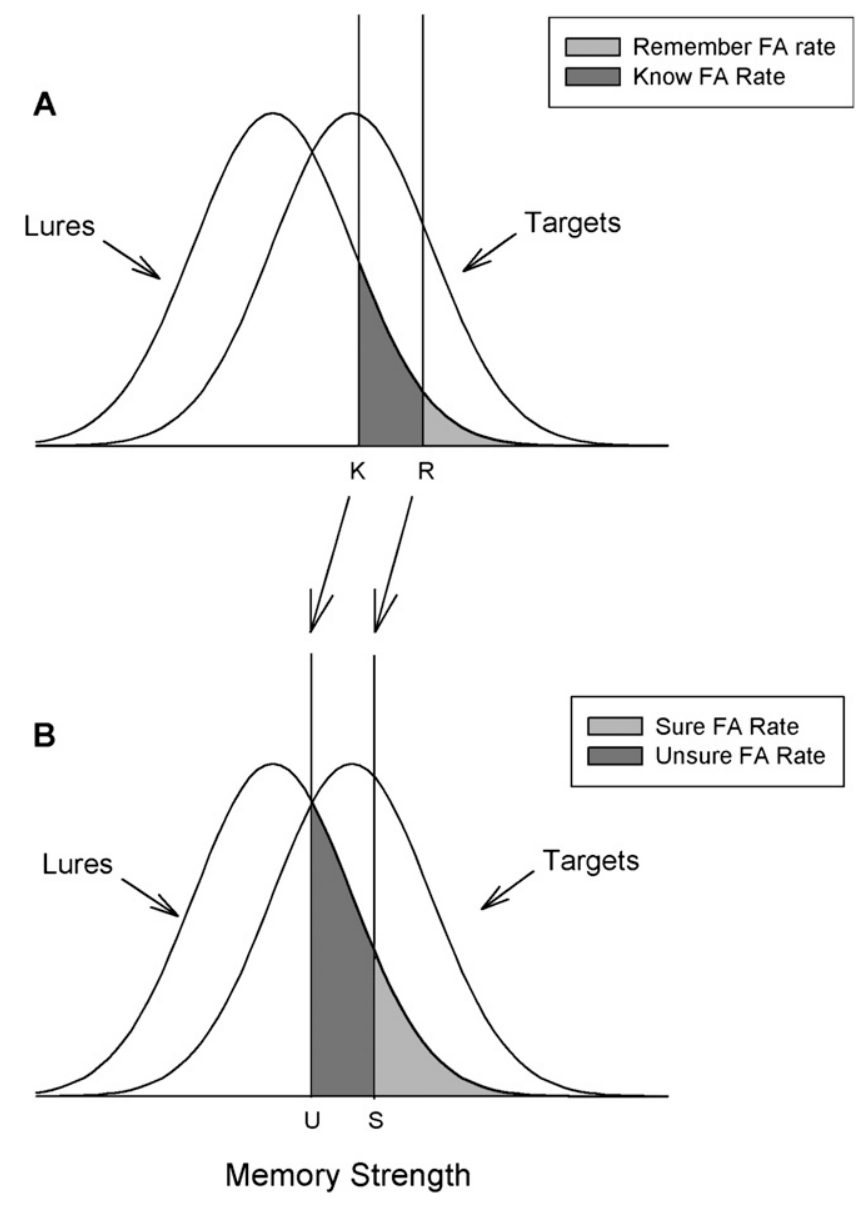

Figure 3. Signal-detection interpretation of the $(A)$ Remember/Know vs. (B) Sure/Unsure dissociation reported by Gardiner and Java (1990), with false alarm rates illustrated by shading. In the upper panel, $\mathrm{K}$ and $\mathrm{R}$ denote the Know and Remember criteria, and in the lower panel $\mathrm{U}$ and $\mathrm{S}$ denote the Unsure and Sure criteria. FA = false alarm rate.

lure distribution, as computed in the standard manner from the overall hit and false alarm rates, is $1 \mathrm{SD}$. The overall hit and false alarm rates in the Sure/Unsure condition were higher (0.66 and 0.29 , respectively), but $d^{\prime}$ remained $\sim 1.0$. Thus, accuracy across the two experiments did not differ.

To understand the crossover effect with respect to hit rates, it is important to first understand the signal-detection interpretation of the false alarm rates in the two conditions, which are higher for Sure/Unsure responses than for Remember/Know responses. The fact that the signal detection analysis provides a clear conceptualization of both Remember and Know false alarm rates is one important way in which it differs from the process-pure interpretation of Remember/Know judgments, which has little to say about Remember false alarm rates in particular. From a signal detection standpoint, the higher false alarm rates in the Sure/ Unsure condition means that the Sure/Unsure decision criteria are placed at a more liberal position on the memory strength axis than are the Remember/Know criteria. This makes sense given that typical Remember/Know instructions indicate that subjects should respond "Old" only if they are confident that they saw the word on the list (i.e., those instructions should induce a relatively conservative placement of the decision criteria). Whatever the reason for the false alarm rate difference, it is clear that a difference exists, and this fact by itself is not problematic for the signal detection account (i.e., there is no reason why the Remember and 
Know criteria must be placed at the exact same point on the memory strength axis as the Sure and Unsure criteria).

In Figure 3A, the Remember and Know criteria (R and $\mathrm{K}$, respectively) are placed in positions that predict Remember and Know false alarm rates that correspond to what was observed in Gardiner and Java's (1990) study. As positioned, the Remember false alarm rate would be 0.03 and the Know false alarm rate would be 0.11 (just as in the observed data). These two false alarm rates are illustrated by the light and dark gray shaded areas, respectively. Figure 3B shows the same signal-detection model except that the Sure and Unsure criteria ( $\mathrm{S}$ and $\mathrm{U}$, respectively) are shifted in lockstep to a more liberal setting. That is, the only difference between the upper and lower panels is that, in the lower panel, the two criteria are both shifted to the left by $0.58 \mathrm{SD}$ units in order to accommodate the higher false alarm rates for Sure and Unsure responses. The predicted Sure and Unsure false alarm rates $(0.10$ and 0.21 , respectively) are again illustrated by the light and dark gray shaded areas, respectively, and they are close to the observed values (0.07 and 0.22, respectively). Although the Sure/Unsure false alarm rates are higher than the corresponding Remember/ Know false alarm rates in both the predicted and the observed data, no crossover is apparent. That is, in both the predicted and the observed data, the Remember and Sure false alarm rates are lower than their corresponding Know and Unsure false alarm rates.

Given that the false alarm rate differences between the Sure/ Unsure condition and the Remember/Know condition can be readily understood in terms of a criterion shift, what does signal detection theory predict about the relevant hit rates as a result of that criterion shift? Figure 4 shows the same illustration as Figure 3 except that now the areas corresponding to the hit rates are shaded. Although the light gray area in the upper panel corresponding to the Remember hit rate is relatively small (smaller than the area corresponding to the Know hit rate), the light gray area corresponding to the Sure hit rate is quite large (and is larger than the area corresponding to the Unsure hit rate). In other words, shifting the criteria to the left produces a crossover pattern for hits. The predicted Remember and Know hit rates are 0.19 and 0.28 , respectively, which are close to the observed values that were 0.18 and 0.30 , respectively. By contrast, the predicted Sure and Unsure hit rates are directionally opposite and are equal to 0.38 and 0.31 , respectively (close to the observed values of 0.39 and 0.27 , respectively). In other words, once the different false alarm rates are taken into consideration, the simplest possible signal-detection analysis predicts the observed dissociation. Note that this interpretation not only provides an account of the counterintuitive dissociation for hits, it also provides an interpretation of the absence of such a dissociation for false alarms, which has heretofore been left unexplained.

All of these predictions were based on an equal-variance signal-detection model, but much evidence suggests that the standard deviation of the target distribution is usually greater than that of the lure distribution (Ratcliff et al. 1992). In agreement with that well-established result, the correspondence between the obtained data shown in Figure 2 and the predicted values shown in Figure 4 can be improved even further by setting the standard deviation of the target distribution to $\sim 1.1$ times that of the lure distribution. And the fit could still be improved more by allowing the distance between the Remember and Know criteria to differ from the distance between the Sure and Unsure criteria. However, the fit is already so close that adding additional parameters, no matter how reasonable they might be, is not necessary. The key points are that the signal-detection model predicts the observed dissociation-one that is often taken as evidence against the model-and that this prediction emerges even when minimal assumptions are made.

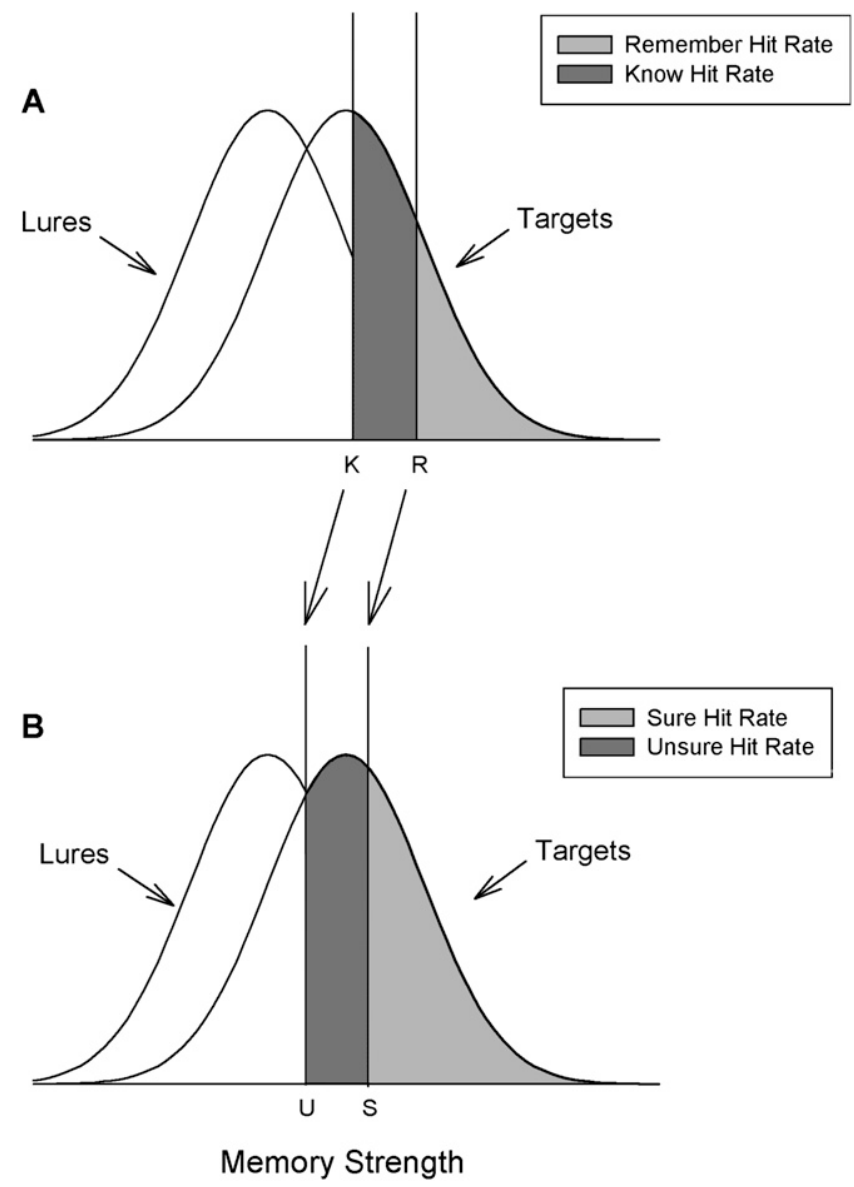

Figure 4. Identical illustration as that shown in Figure 3 except that hit rates (instead of false alarm rates) are illustrated by shading.

I turn now to a consideration of an example from the neuroscience literature. Verfaellie et al. (2008) recently used the Remember/Know procedure with amnesic patients and controls in an effort to determine which memory process (recollection or familiarity) is more impaired in amnesia. Participants in that experiment studied three kinds of items during list presentation: items presented once, items presented three times with the same context word each time, and items presented three times with a different context word each time. The context words were semantically related to the study word, and the hypothesis was that repetition would enhance recollection and familiarity in controls (both same context and varied context), whereas it would mainly enhance familiarity in amnesic patients. The Remember/ Know procedure was used to test this prediction.

As is commonly done, the authors considered the Remember hit rate to provide an estimate of recollection, and they computed a familiarity estimate for each group using the standard Independence Remember-Know method (according to which familiarity equals the Know hit rate divided by the quantity 1 minus the Remember hit rate). Using this method, repetition was found to significantly increase recollection in controls only, but repetition significantly increased familiarity in both groups. This result is consistent with other evidence suggesting that recollection is selectively impaired in amnesia. However, the important point is that this interpretation is dependent on the process-pure interpretation of Remember/Know judgments and does not apply if the signal-detection interpretation is correct. Verfaellie et al. (2008) briefly noted that their results do not seem compatible 
with signal-detection theory, but they did not present the signal-detection account of their data and ask how well it can describe their results.

To perform a signal-detection analysis, a Gaussian model is fit to the Remember/Know data. The data to be fit in this case include three Remember hit rates (one for each repetition condition), three corresponding Know hit rates, one Remember false alarm rate, and one Know false alarm rate. The mean and standard deviation of the lure distribution are arbitrarily set to 0 and 1 , respectively, and the other parameters are scaled with respect to those values. The parameters to be estimated include the means of the three target distributions, their standard deviations (which, for simplicity, were assumed to be equal to each other), and the locations of both the Remember and the Know criteria. These parameters are then adjusted using an optimization algorithm (e.g., Excel's "solver" function) until the optimal parameter estimates are obtained (e.g., until the squared deviations between predicted and obtained hit and false alarm rates are minimized).

Figure 5A shows the signal-detection interpretation (i.e., the signal-detection model) that corresponds most closely to the amnesic data reported by Verfaellie et al. (2008); Figure 5B shows the interpretation that corresponds most closely to their control data. In both cases, the parameters that define the target distributions and that specify the placement of the Remember and Know criteria were estimated in the manner described above. The observed and predicted hit and false alarm rates are shown in Table 1, and the fact that they are in close agreement is readily apparent.

For the amnesic patients, the target and lure distributions were estimated to have approximately equal variance (a typical result for weak memory conditions and for amnesic patients in particular). For the controls, the target distribution was estimated to have greater variance than the lure distribution (the typical result in strong memory conditions). Interpreted in terms of signal detection theory, the results simply indicate that repetition increased the mean and variance of the memory strength distribu-

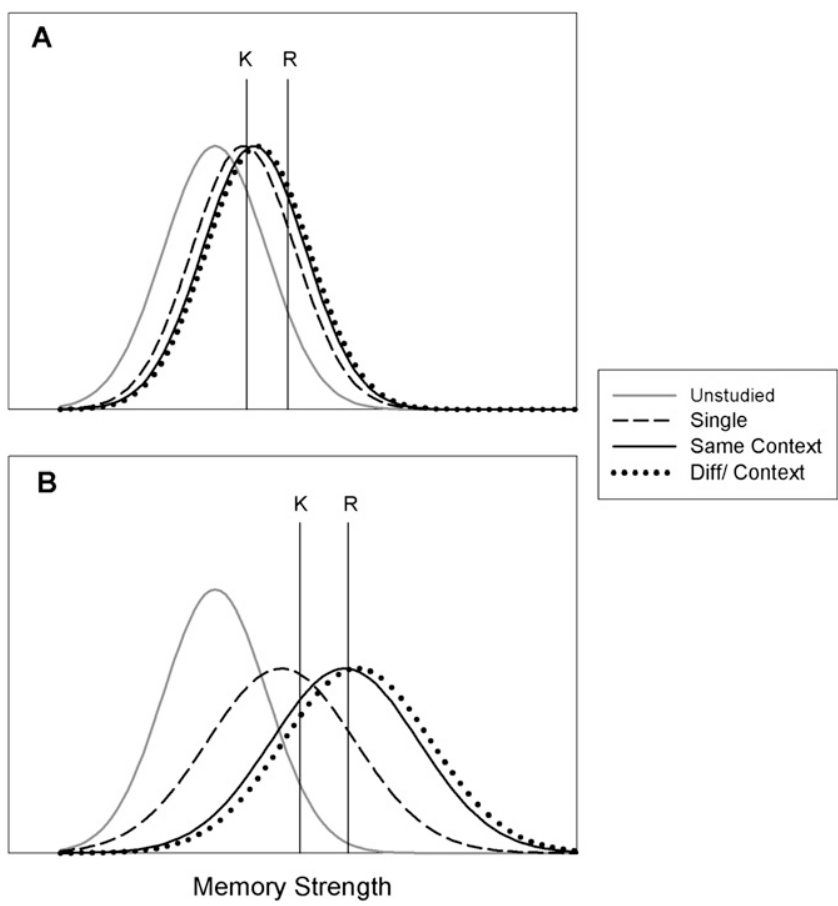

Figure 5. Signal-detection interpretation of Remember/Know data for $(A)$ amnesics and $(B)$ controls, reported by Verfaellie et al. (2008). tions for controls more so than for the patients (not that familiarity was selectively affected in amnesia). That the variance of the target distribution would increase with its mean is a sensible result (e.g., it is an extremely common statistical result across domains) and one that is commonly observed in studies of recognition memory (e.g., see Glanzer et al. 1999 for a review). Further, the fact that, in absolute terms, repetition increased the mean (and variance) of the target distributions to a lesser extent for the patients than for the controls is also easily understood, in light of the fact that the patients are memory impaired. In relative terms, the results for the two groups were quite similar. Specifically, varying semantic context across three stimulus repetitions increased $d^{\prime}$ by about $10 \%$ in both amnesics and controls.

The point is not that the signal detection model offers the superior interpretation of these data or that the interpretation offered by Verfaellie et al. (2008) is necessarily wrong. In fact, as is often true, these Remember/Know data do not have sufficient power to discriminate between competing models, and they do not strongly constrain the signal-detection parameters estimated above. Instead, the point is that a viable alternative to the processpure interpretation exists, and it is more compelling than is generally appreciated in the neuroscience literature. Remember/Know data that are equally interpretable in terms of a process-pure account and in terms of signal-detection theory should probably not be presented as if they are selectively interpretable in terms of the former. Moreover, if one intends to argue against it, the only way to fairly evaluate the signal-detection interpretation is to illustrate the detection model that best accounts for the data. When that is done for the Remember/Know data reported by Gardiner and Java (1990), as well as for the Remember/Know data reported by Verfaellie et al. (2008), it becomes clear that the signaldetection alternative offers a viable and parsimonious account.

Although fitting the signal-detection model to Remember/ Know data is an important step to take before declaring its inability to explain the data, it seems reasonable to assume that the model will be found to provide an acceptable fit in the large majority of studies performed in the future because this has proven to be true of the large majority of studies performed in the past (e.g., Dunn 2004, 2008). Thus, as an alternative to model fitting, another reasonable (and simpler) strategy would be to design studies in such a way as to avoid the strength confound that is highlighted by the signal-detection account. In general, the Remember/Know procedure is used in the neuroscience literature to either directly quantify recollection and familiarity (e.g., so that the effects of lesions on these two processes can be measured) or to separate recollection-based from familiarity-based recognition decisions (e.g., so that correlated brain activity can be measured using fMRI). If the signal-detection model is correct, these strategies would not be valid. What strategies would be?

If recollection and familiarity are aggregated into the memory strength signal (as a dual-process version of signal-detection theory assumes they are), there would be no obvious way to extract precise quantitative estimates of the two processes from behavioral data. However, even in the absence of quantitative estimates, the signaldetection account suggests useful strategies for identifying the brain structures that subserve recollection and familiarity. The key methodological refinement would be to equate Remember judgments and Know judgments for strength, thereby eliminating the strength confound that plagues the Remember/Know procedure as it is typically used. To do so, confidence ratings can be used in conjunction with the Remember/Know procedure. In the behavioral literature, for example, Rotello and Zeng (2008) asked for both Remember/Know judgments and confidence ratings for each item that was declared to be old. Although most Remember judgments are made with higher confidence than Know judgments, as the signal-detection account requires, some Remember judgments are 
Table 1. Observed and predicted hit rates, false alarm rates, and Remember/Know familiarity estimates for data reported by Verfaellie et al. (2008)

\begin{tabular}{|c|c|c|c|c|c|}
\hline \multirow[b]{2}{*}{ Condition } & & \multicolumn{2}{|c|}{ Amnesic } & \multicolumn{2}{|c|}{ Control } \\
\hline & & Predicted & Observed & Predicted & Observed \\
\hline Single & $\begin{array}{l}\text { Overall HR } \\
\text { Remember HR } \\
\text { Know HR } \\
\text { IRK } \\
d^{\prime}\end{array}$ & $\begin{array}{l}0.48 \\
0.18 \\
0.30 \\
0.36 \\
0.56\end{array}$ & $\begin{array}{l}0.46 \\
0.20 \\
0.26 \\
0.36 \\
0.51\end{array}$ & $\begin{array}{l}0.40 \\
0.18 \\
0.22 \\
0.27 \\
1.39\end{array}$ & $\begin{array}{l}0.40 \\
0.18 \\
0.22 \\
0.27 \\
1.39\end{array}$ \\
\hline Same-context repetition & $\begin{array}{l}\text { Overall HR } \\
\text { Remember HR } \\
\text { Know HR } \\
\text { IRK } \\
d^{\prime}\end{array}$ & $\begin{array}{l}0.55 \\
0.24 \\
0.32 \\
0.42 \\
0.75\end{array}$ & $\begin{array}{l}0.57 \\
0.23 \\
0.34 \\
0.44 \\
0.79\end{array}$ & $\begin{array}{l}0.73 \\
0.48 \\
0.25 \\
0.48 \\
2.25\end{array}$ & $\begin{array}{l}0.73 \\
0.48 \\
0.25 \\
0.50 \\
2.26\end{array}$ \\
\hline Different-context repetition & $\begin{array}{l}\text { Overall HR } \\
\text { Remember HR } \\
\text { Know HR } \\
\text { IRK } \\
d^{\prime}\end{array}$ & $\begin{array}{l}0.59 \\
0.26 \\
0.32 \\
0.44 \\
0.83\end{array}$ & $\begin{array}{l}0.60 \\
0.26 \\
0.34 \\
0.47 \\
0.87\end{array}$ & $\begin{array}{l}0.78 \\
0.55 \\
0.23 \\
0.52 \\
2.43\end{array}$ & $\begin{array}{l}0.78 \\
0.55 \\
0.23 \\
0.53 \\
2.42\end{array}$ \\
\hline Nonstudied & $\begin{array}{l}\text { FAR } \\
\text { Remember-FAR }\end{array}$ & $\begin{array}{l}0.27 \\
0.08\end{array}$ & $\begin{array}{l}0.27 \\
0.08\end{array}$ & $\begin{array}{l}0.05 \\
0.01\end{array}$ & $\begin{array}{l}0.05 \\
0.01\end{array}$ \\
\hline
\end{tabular}

$\mathrm{HR}=$ hit rate; $\mathrm{FAR}=$ false alarm rate; $\mathrm{IRK}=$ Remember-Know familiarity estimate, calculated using the independence method; and $d^{\prime}=z$-transformed hit rate minus $z$-transformed false alarm rate.

pure familiarity-based experience of strong recognition is relatively rare because, usually, strong familiarity is accompanied by recollection. This is consistent with the finding that high-confidence recognition decisions are only occasionally associated with Know judgments (as discussed above) and are instead usually associated with Remember judgments (e.g., Wixted and Stretch 2004). However, if hippocampal lesions impair recollection while leaving familiarity preserved, highconfidence recognition in the absence of recollection (i.e., high-confidence Know decisions) should be a more common experience in hippocampal patients than in matched controls. That is, the patients should not only experience the relatively rare high-confidence familiarity-based recognition that controls occasionally experience, but they should experience the high-confidence familiaritybased recognition that controls would have experienced had recollection not so often accompanied a strong sense of familiarity. Thus, if the hippocampus selectively subserves recollection, then hippocampal patients should report high-confidence Know judg-

made with lower levels of confidence, and some Know judgments are made with higher levels of confidence. This can occur because the location of the Remember criterion on the memory strength axis exhibits trial-to-trial variability with respect to the confidence criteria. For example, the Remember criterion (Fig. 1B) might be placed somewhat below the criterion for a confidence rating of 6 (Fig. 1A) on some trials and well above it on others. Much evidence suggests that the Remember criterion does, indeed, exhibit such trial-to-trial variability, and this explains why, for example, Know judgments can sometimes be associated with high confidence (e.g., Wixted and Stretch 2004; Rotello et al. 2005; Starns and Ratcliff 2008). Rotello and Zeng (2008) found that reaction time distributions associated with Remember and Know judgments were very similar once confidence was equated (i.e., the usual speed advantage for Remember judgments all but disappeared).

Using the standard Remember/Know procedure, it has been reported that hippocampal activity, as measured by fMRI, is elevated at retrieval for Remember judgments but not for Know judgments (e.g., Eldridge et al. 2000). This finding is analogous to the finding in the behavioral literature that Remember reaction times are faster than Know reaction times when the standard Remember/Know procedure is used. However, the reaction time difference disappears when Remember judgments and Know judgments are equated for strength, and the question of interest is whether the same would be true of hippocampal activity. This question has not been empirically addressed, but it could be. If hippocampal activity remained selectively elevated for Remember judgments even when Remember and Know judgments were equated for strength, it would be consistent with the idea that the hippocampus selectively subserves recollection. However, if hippocampal activity were instead found to be elevated for both Remember and Know judgments when they are equated for strength, it would be consistent with the idea that the hippocampus subserves declarative memory in general, whether such memory is recollection or familiarity based.

A related approach could be used to test the effects of hippocampal lesions on Remember/Know judgments. For example, unimpaired individuals occasionally experience high-confidence recognition in the absence of recollection (as when a face seems extremely familiar even when it cannot be placed). However, the ments more often than controls. This prediction has never been tested, but it is the kind of informative test that is suggested by a dual-process version of signal-detection theory.

Determining whether recent findings in the neuroscience literature hold, even when Remember/Know judgments are equated for strength, should be a high priority. Many studies that have relied on the process-pure interpretation of Remember/Know judgments have been taken to suggest that the hippocampus selectively supports the recollection process. However, when those same results are interpreted in terms of the signal-detection model, they instead suggest that the hippocampus supports strong memories, whether those memories are based on recollection or on familiarity (Squire et al. 2007). One way to advance this line of inquiry without becoming mired in the debate over the interpretation of Remember/Know judgments would be to equate them for strength.

\section{References}

Aggleton JP, Vann SD, Denby C, Dix S, Mayes AR, Roberts N, Yonelinas AP 2005. Sparing of the familiarity component of recognition memory in a patient with hippocampal pathology. Neuropsychologia 43: 1810-1823.

Algarabel S, Pitarque A. 2007. Context, remember-know recognition judgements, and ROC parameters. Memory 15: 477-494.

Benjamin AS. 2005. Recognition memory and introspective remember/ know judgments: Evidence for the influence of distractor plausibility on "remembering" and a caution about purportedly nonparametric measures. Mem Cognit 33: 261-269.

Dewhurst SA, Holmes SJ, Brandt KR, Dean GM. 2006. Measuring the speed of the conscious components of recognition memory: Remembering is faster than knowing. Conscious Cogn 15: 147-162.

Donaldson W. 1996. The role of decision processes in remembering and knowing. Mem Cognit 24: 523-533.

Dunn JC. 2004. Remember-Know: A matter of confidence. Psychol Rev 111: 524-542.

Dunn JC. 2008. The dimensionality of the remember-know task: A statetrace analysis. Psychol Rev 115: 426-446.

Egan JP. 1958. Recognition memory and the operating characteristic. Technical note no. AFCRC-TN-58-51. Hearing and Communication Laboratory, Indiana University, Bloomington, IN.

Eldridge LE, Knowlton BJ, Furmanski C, Bookheimer S, Engel SA. 2000. Remembering episodes: A selective role for the hippocampus during retrieval. Nat Neurosci 3: 1149-1152.

Eldridge LL, Engel SA, Zeineh MM, Bookheimer SY, Knowlton BJ. 2005. A dissociation of encoding and retrieval processes in the human hippocampus. J Neurosci 25: 3280-3286. 
Gardiner JM, Java RI. 1990. Recollective experience in word and nonword judgments. Mem Cognit 18: 23-30.

Glanzer M, Kim K, Hilford A, Adams JK. 1999. Slope of the receiveroperating characteristic in recognition memory. J Exp Psych Learn Mem and Cogn 25: 500-513.

Gonsalves BD, Kahn I, Curran T, Norman KA, Wagner AD. 2005. Memory strength and repetition suppression: Multimodal imaging of medial temporal cortical contributions to recognition. Neuron 47: 751-761.

Hirshman E, Henzler A. 1998. The role of decision processes in conscious recollection. Psych Sci 9: 61-65.

Holdstock JS, Mayes AR, Isaac CL, Cezayirli E, Roberts N, O’Reilly R, Norman K. 2002. Under what conditions is recognition spared relative to recall after selective hippocampal damage in humans? Hippocampus 12: $341-351$.

Holdstock JS, Mayes AR, Gong Q, Roberts N, Kapur N. 2005. Item recognition is less impaired than recall and associative recognition in a patient with selective hippocampal damage. Hippocampus 15: 203-215.

Inoue C, Bellezza FS. 1998. The detection model of recognition using know and remember judgments. Mem Cognit 26: 299-308.

Mandler G. 1980. Recognizing: The judgment of previous occurrence. Psychol Rev 87: 252-271.

Moscovitch D, McAndrews MP. 2002. Material-specific deficits in remembering in patients with unilateral temporal lobe epilepsy and excisions. Neuropsychologia 40: 1335-1342.

Ratcliff R, Sheu CF, Gronlund SD. 1992. Testing global memory models using ROC curves. Psychol Rev 99: 518-535.

Rotello CM, Zeng M. 2008. Analysis of RT distributions in the rememberknow paradigm. Psychon Bull Rev 15: 825-832.
Rotello CM, Macmillan NA, Reeder JA, Wong M. 2005. The remember response: Subject to bias, graded, and not a process-pure indicator of recollection. Psychon Bull Rev 12: 865-873.

Squire LR, Wixted JT, Clark RE. 2007. Recognition memory and the medial temporal lobe: A new perspective. Nat Rev Neurosci 8: $872-883$.

Starns JJ, Ratcliff R. 2008. Two dimensions are not better than one: STREAK and the univariate signal detection model of remember/know performance. J Mem Lang 59: 169-182.

Tulving E. 1985. Memory and consciousness. Can Psychol 26: 1-12.

Uncapher MR, Rugg MD. 2005. Encoding and the durability of episodic memory: A functional magnetic resonance imaging study. J Neurosci 25 : 7260-7267.

Verfaellie M, Rajaram S, Fossum K, Williams L. 2008. Not all repetition is alike: Different benefits of repetition in amnesia and normal memory. $J$ Int Neuropsychol Soc 14: 365-372.

Wixted JT. 2007. Dual-process theory and signal-detection theory of recognition memory. Psychol Rev 114: 152-176.

Wixted JT, Stretch V. 2004. In defense of the signal detection interpretation of Remember/Know judgments. Psychon Bull Rev 11: 616-641.

Yonelinas AP. 2002. The nature of recollection and familiarity: A review of 30 years of research. J Mem Lang 46: 441-517.

Yonelinas AP, Kroll NE, Quamme JR, Lazzara MM, Sauve MJ, Widaman KF, Knight RT. 2002. Effects of extensive temporal lobe damage or mild hypoxia on recollection and familiarity. Nat Neurosci 5: 1236-1241.

Received December 17, 2008; accepted in revised form March 13, 2009. 


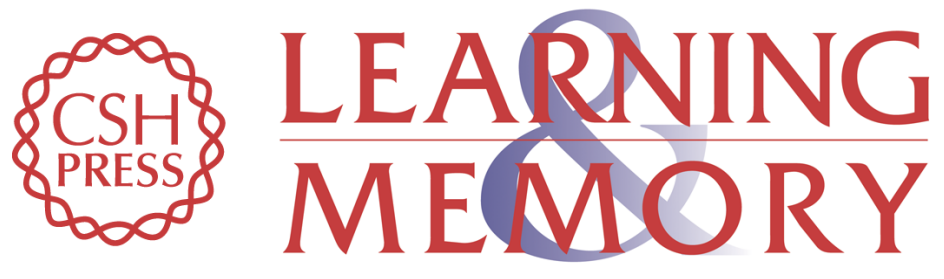

\section{Remember/Know judgments in cognitive neuroscience: An illustration of the underrepresented point of view}

John T. Wixted

Learn. Mem. 2009, 16:

Access the most recent version at doi:10.1101//m.1312809

References This article cites 30 articles, 2 of which can be accessed free at: http://learnmem.cshlp.org/content/16/7/406.full.html\#ref-list-1

License

Email Alerting

Receive free email alerts when new articles cite this article - sign up in the box at the Service top right corner of the article or click here. 